with chromosome deletion 17p are described for the present series of patients and those in the literature: malformed fingers, congenital heart-defect, sacral dimple, cryptorchidism, and malformed kidneys. (De Rijk-van Andel JF et al. Diagnostic features and clinical signs of 21 patients with lissencephaly type I. Dev Med Child Neurol Aug 1990; 32:707-717).

COMMENT. The diagnosis of lissencephaly is made by clinical and neuroradiological findings. Cases of isolated lissencephaly may be distinguished from the Miller-Dieker syndrome without the results of chromosome analysis in most cases. The CT signs of lissencephaly include smooth brain surface and no Sylvian fissure with figure 8 appearance, sharp demarcation between gray and white matter, and colpocephaly. Facial dysmorphism may show high forehead, bitemporal hollowing, and micrognathia.

\title{
SCHIZENCEPHALY AND BEHAVIORAL CORRELATES
}

The MRI appearance and neuropsychologic and speech/language evaluations in three patients with schizencephaly are described from the Department of Radiology, Michigan State University, East Lansing, MI. All patients presented with a seizure disorder and they were left-handed without familial sinistrality in first degree relatives. The primary areas of cerebral involvement were the left parasylvian and pararolandic regions with varying degrees of secondary involvement of the right hemisphere. There was mild right-sided limb hypoplasia, and motor dexterity with the dominant left hand was better than with the right hand. Two patients showed significant impairment in finger localization and tactile form recognition particularly with the nondominant right hand. The level of general intellectual functioning related to the amount of brain tissue involved, and neurobehavioral abilities reflected the location of the brain malformation and the prenatal onset of the disorder. The full scale IQ on the WAIS-R ranged from a low of 65 to a high of 87 . Only one patient showed a higher verbal IQ than performance IQ and her visuospatial construction abilities and visual memory abilities were significantly impaired. There were varying degrees of linguistic deficit with relatively greater difficulties in syntactical speech than in semantic aspects. (Aniskiewicz AS et al. Magnetic resonance imaging and neurobehavioral correlates in schizencephaly. Arch Neurol August 1990; 47:911-916).

COMMENT. Yakovlev and Wadsworth first described the schizencephalies as congenital defects in the cerebral mantle in 1946. The patients in the present study shared features in common with the pathologic left handedness syndrome of Orsini and Satz; predominantly left-sided cerebral lesion with onset before six years of age and involving speech/language areas of frontotemporal parietal cortex, atypical or right-sided hemispheric speech representation, impaired visuospatial abilities and preserved verbal cognitive abilities, right limb hypoplasia, right hand motor impairment, and absence of familial sinistrality. (Orsini DL, Satz P. A syndrome of pathological 
left-handedness: Correlates of early left hemisphere injury. Arch Neurol 1986; $43: 333-337$ ).

\section{SEIZURE DISORDERS}

PHENYTOIN FOR POST-TRALMATIC SEIZURES

A randamized, double-blind study of phenytoin was conducted in 404 patients with serious head trauma at the Departments of Neurological Surgery, Rehabilitation Medicine and Medicine, University of Washington, Seattle. An intravenous loading dose of phenytoin was given within 24 hours of injury to 208 patients and 196 received placebo for a one year period in a double-blind fashion. Serum levels were maintained in the high therapeutic range (3-6 mamol/l). Statistical analyses were performed according to the intention to treat and based on efficacy. Between the initial drug loading dose and day seven, $3.6 \%$ of patients assigned to phenytoin had seizures compared to $14 \%$ of patients assigned to placebo $(P<0.001)$. From day eight to the end of year one and the end of year two of the study, there was no significant difference between the seizure incidence in the phenytoin and placebo groups, approximately 1 in 5 having a recurrence. The relapse was not explained by low phenytoin levels. (Temkin NR et al. A randomized, double-blind study of phenytoin for the prevention of post-traumatic seizures. $\quad \underline{N}$ Engl J Med August 23, 1990; 323:497-502).

COMMENT. The authors concluded that phenytoin exerts a beneficial effect by reducing posttraumatic seizures only during the first week after severe head injury. Dr. Allen Hauser, in an editorial comment, states that early administration of loading doses of IV phenytoin to patients with severe head injury may be warranted to prevent early seizures and their complications, but prolonged therapy after stabilization does not seem justified. Other anticonvulsants such as phenobarbital and benzodiazepines should be considered as alternatives, and treatment with antioxidants which reduce edema and prevent neuronal damage caused by iron salts deposited at the time of injury may be of benefit.

FETAL ANTIOONUULSANT SYNDROME WITH NEOCEREBELLLAR HYPOPLASIA

An infant with dysmorphic features and hypoplasia of the cerebral hemispheres and cerebellum is reported from the John Radcliffe Hospital, Oxford, England as an extreme example of anticonvulsant teratogenicity. The mother was epileptic and she had taken phenytoin and sodium valproate throughout pregnancy. The infant was cyanosed and hypotonic at birth with Apgar scores of 4 at one minute and 6 at five minutes. She had abnormalities of the toes, fingers, nails, elbows, hips, ears, and an antimongoloid slant to the eyes with hypertelorism. Intractable seizures began ten minutes after delivery and she died at 66 hours of age. Postmortem neuropathological examination showed a thickened skull, recuced size of the pons and neocerebellum and 